\title{
REFLEXIONES DE E. SCHRÖDINGER SOBRE CAUSALIDAD, INDETERMINISMO Y LIBRE
}

\author{
ALBEDRÍO \\ (E. Schrödinger's Reflections on Causation, \\ Determinism and Freewill)
}

\author{
Josefa Castellà Cid.
}

\section{I.E.S Roquetes, Tarragona}

\begin{abstract}
Resumen: La admiración que generó el éxito del sistema newtoniano llevó al intento de extender el modelo determinista de causalidad a todos los dominios del conocimiento empírico, tanto de las ciencias naturales como de las ciencias humanas y sociales. Pero este intento plantea cuestiones como las siguientes: ¿es posible predecir el comportamiento de un sistema cuántico? ¿Es posible conciliar el determinismo y el libre albedrío? Las diversas respuestas que E.Schrödinger ofrece a estas preguntas invitan a la reflexión.

Palabras clave: causalidad, azar, determinismo, indeterminismo, libre albedrío.

Abstract: The high success of the Newtonian determinist system produced great admiration and gave the impression that the determinist model is ideal for scientific explanation. So scientists wished to extend the determinist causation model to all the domains of the empirical knowledge, including natural, human and social sciences. But this attempt presents multiple questions: is it posible, for instance, to predict the behaviour of a quantic system? Is it posible to reconcile determinism with free will? Schrödinger's several contributions on these topics rose our reflection.

Keywords: causality, chance, determinism, indeterminism, free will.

Recibido: 27/03/2016. Aprobado: 9/4/2016
\end{abstract}




\section{Introducción}

El ser humano intenta entenderlo todo, tanto el mundo físico que se extiende a su alrededor como el mundo psíquico que intuye en su interior, tanto la res extensa como la res cogitans. Podemos pensar que, para hacer inteligible el mundo material, creó la noción de causalidad, con la cual conectaba unos hechos - a los que consideraba efectos- con otros hechos precedentes, que de alguna manera los producían y los explicaban - y a los que consideraba causas-. Y para referirse a hechos que no podía conectar con unas supuestas causas, es decir, a situaciones a las que no podía aplicar la relación de causalidad, creó el concepto de casualidad o azar.

A partir de nociones del lenguaje común como la causalidad y la casualidad, el científico fue más allá y otorgó a los dominios de la Naturaleza a los que podía aplicar la relación de causalidad una interpretación determinista, con la cual suponía que existen leyes naturales necesarias que determinan unívocamente los estados de un sistema a partir de otros estados anteriores. $\mathrm{Y}$ a aquellos campos a los que no podía aplicar la relación de causalidad les dio una interpretación indeterminista, según la cual los hechos físicos no tienen causas anteriores que los expliquen necesariamente, porque no existen leyes necesarias que determinen unívocamente los estados de un sistema a partir de otros estados anteriores. Así, bajo una perspectiva indeterminista solo es posible establecer leyes probabilísticas.

Por otra parte, el ser humano para entender el mundo psíquico, creó nociones como la conciencia, la responsabilidad y la libertad. Observó su mundo interior y le pareció que, cuando no se veía coaccionado, se sentía 
libre y podía decidir lo que iba a hacer, según sus propios deseos. Entonces se sentía responsable de sus decisiones y de sus acciones. El filósofo designó esta situación con la expresión "libre albedrío". Le pareció que los seres que no eran humanos no disponían de esta facultad de decidir por sí mismos ${ }^{1}$. Y estuvo satisfecho de disponer de una capacidad de la que no disfrutaban otros seres.

Pero el ser humano, que pretende comprenderlo todo, fue más allá. Miró más detenidamente a las personas de su alrededor y creyó que podía establecer conexión entre sus pensamientos, voliciones, decisiones y actuaciones y otros hechos anteriores, de manera que estos se pudieran considerar causas de aquellos. Así pues, aplicó el concepto de causa no solo a la res extensa sino también a la res cogitans, a pesar de que esta parecía disfrutar de una facultad de la que aquella carecía, el libre albedrío ${ }^{2}$. Sospechó que bajo la aparente libertad corría un río subterráneo de influencias inconscientes, que podían salir a la luz de la conciencia en cualquier momento. Y cuando descubría las causas de las que provenían los fenómenos anímicos, el mundo psíquico parecía tan determinado como el mundo físico de la mecánica newtoniana, sistema determinista que era ampliamente admirado por el gran éxito que había alcanzado en dominios como la astronomía. Así, la admiración que generó el modelo de Newton aplicado al sistema solar hizo pensar que dicho

${ }^{1}$ Esta concepción filosófica tradicional plantea la cuestión de si los animales disponen de cierto libre albedrío, de qué noción de libertad se les puede aplicar.

${ }^{2}$ Como pensaba Descartes, por lo cual Arana escribe: "El primer rasgo de la libertad humana, tal como la concibe Descartes, es que solo se detecta en una de las dos dimensiones en que el mundo es dividido por la conciencia: la interior"(Arana, 2005: 24) 
modelo determinista proporcionaba el ideal de explicación científica válido para todas las ramas del saber.

De esta manera el determinismo se extendió también a las ciencias humanas y sociales, extensión que obedecía a la tendencia natural del ser humano a reducir sus conceptos generales, sus principios y sus esquemas explicativos, a integrar en una unidad todos los fenómenos, aunque fueran de muy diversa índole, a seguir un principio de economía que le evitara malgastar su energía mental y le permitiera aprovecharla al máximo para comprender el mayor número posible de fenómenos con el mínimo número posible de nociones, buscando un ideal de sencillez y claridad. Por esta razón, el ser humano tiende a unificar todas las nociones que ha creado para entender el mundo, tanto las que utiliza para hacer inteligible la res extensa como la res cogitans.

Así, la aplicación de la noción de causa al dominio de la res cogitans, tradicionalmente caracterizado por la libertad, plantea un problema: ¿cómo armonizar la conciencia que normalmente tiene el ser humano de ser libre y responsable con la existencia de causas que condicionan sus pensamientos, sentimientos, deseos y decisiones?

En la actualidad el antiguo problema del libre albedrío se ha acentuado con el vertiginoso avance de las ciencias de la vida, del hombre y de la sociedad, con los continuos y sorprendentes descubrimientos de la genética, la neurociencia, la sociología... cuyos investigadores cada día nos desvelan nuevos factores que condicionan nuestra conducta y nuestra psique. Así, con el progreso de estas ciencias empíricas los supuestos deterministas se han hecho cada vez más comunes y aceptamos implícitamente que nuestros rasgos 
psíquicos están fuertemente influidos por condicionantes genéticos, neurológicos, familiares, sociales...

Schrödinger, físico y humanista, creador de la ecuación — que ha sido interpretada de forma determinista ${ }^{3}$ - de la función de onda $\Psi$ - que, en cambio, ha sido interpretada de forma probabilística ${ }^{4}$, se interesó por los problemas que presentan la causalidad y el determinismo, nociones que, para muchos de los creadores de la mecánica cuántica —como Einstein, Bohr, Heisenberg y Schrödinger-, eran muy cercanas, tan cercanas que dichos científicos consideraban el principio de causalidad como equivalente al principio determinista, propio de la física newtoniana. Heisenberg expresaba esta concepción con las siguientes palabras:

Cuando experimentamos que algo ocurre, presuponemos en todo caso que algo ha precedido a aquella ocurrencia, algo de lo que ella se sigue según una regla. Así fue paulatinamente restringiéndose el alcance del principio de causalidad, hasta resultar equivalente a la suposición de que el acontecer de la Naturaleza está unívocamente determinado, de modo que el conocimiento preciso de la Naturaleza o de cierto sector suyo basta, al menos en principio, para predecir el futuro. Precisamente la Física newtoniana se hallaba estructurada de modo tal que a partir del estado de un sistema en un instante determinado podía preverse el movimiento futuro del sistema (Heisenberg, 1985: 702).

3 Refiriéndose a $\Psi$ Schrödinger manifestaba: "Su ecuación de movimiento, la ley de su evolución temporal, cuando el sistema no es perturbado, no cede en nada, en claridad y precisión, a las ecuaciones de movimiento del modelo clásico” (Schrödinger, 1935: 156).

${ }^{4}$ Así, Schrödinger asumía, en 1930, la interpretación estadística que Born había dado a $\Psi$ (Arana, 2001: 25) 
Así, en este párrafo, se puede percibir cómo Heisenberg encontraba en Kant la adecuada explicación de la noción de causa, tal como veía que la utilizaban la mayoría de los físicos de su época, entre los que se encontraba Schrödinger.

Mas, por otra parte, Schrödinger también pretendía ofrecer una concepción filosófica que integrara las diversas partes del conocimiento, una visión general que ayudara a entender el mundo como un todo y a situar en él al ser humano, puesto que la cuestión esencial a la cual pretendía responder a través de todas sus indagaciones, tanto científicas como filosóficas, era la siguiente: ¿Quién soy yo?, en palabras de Plotino: "Y nosotros, ¿qué somos en el fondo?” (Schrödinger, 1951: 14). Así, con esta motivación, Schrödinger se planteaba también el problema del libre albedrío, se preguntaba si somos tan libres como creemos o si nuestro yo está determinado, puesto que entendía el yo como la mente, como el lienzo (Schrödinger, 1956: 127) que recoge todas nuestras experiencias, presentes y pasadas, las cuales indudablemente actúan como condicionantes de nuestras voliciones y decisiones.

Precisamente la interpretación indeterminista de la mecánica cuántica con la que veremos que Schrödinger simpatizó ${ }^{5}$ - había alimentado la esperanza de justificar la libertad interna del ser humano. Sin embargo, como también veremos, según Schrödinger este intento de justificación solo constituía una vana ilusión que él, con sus análisis, pretendió disipar.

${ }^{5}$ Como muestra en la conferencia titulada “¿Qué es una ley de la Naturaleza?”, pronunciada en 1922. En ella vemos que Schrödinger "ya era indeterminista en cierto sentido antes de que surgiera la tesis del indeterminismo cuántico" (Arana, 2001: 17). 
Comenzaremos mostrando el legado que Schrödinger heredó de Boltzmann y Exner, profesores de la Universidad de Viena, donde inició su carrera como físico. L. Boltzmann fue el primer físico que cuestionó el estricto determinismo (Jammer, 1973: 587) y Exner, su sucesor en la Universidad, propuso una interpretación indeterminista del comportamiento aparentemente determinista de los fenómenos macroscópicos puesto que consideraba que éstos constituían el resultado de una gran cantidad de procesos que en el fondo, a nivel microscópico, eran probabilísticos.

\section{El legado de Boltzmann y Exner}

Schrödinger había heredado de Boltzmann la línea de pensamiento estadístico y atomístico ${ }^{6}$ en la que se mantuvo durante toda su vida ${ }^{7}$. Hablando de Boltzmann, Schrödinger había declarado: "Su línea de pensamiento puede llamarse mi primer amor en ciencia. Nunca nadie así me ha encantado ni volverá a hacerlo de nuevo" (Scott, 1967: 15). Aunque Schrödinger no pudo asistir a sus clases porque había iniciado sus estudios en la Universidad de Viena en 1906, el año en que murió Boltzmann, influyó decisivamente en él

\footnotetext{
${ }^{6}$ Así, Schrödinger siempre defendió la discontinuidad atomista. "El continuo ofrece muchos problemas" escribió (Schrödinger, 2009: 40), pero también escribió: "Al abandonar la discontinuidad no hemos de temer el perder el largo tiempo honorado atomismo" en "The meaning of wave mechanics" (Schrödinger, 1984: 706).

${ }^{7}$ La teoría cuántica, a la que Schrödinger dedicó la mayor parte de su trabajo como físico, había surgido del punto de vista estadístico, manifestaba él mismo al recordar que nació de un problema fundamental de la teoría estadística del calor de Planck (Schrödinger, 1983: 156).
} 
un profesor que había sido discípulo de éste, Exner, del cual Schrödinger adquirió las bases para su trabajo posterior. Así, la mayoría de los primeros trabajos de Schrödinger estuvieron dedicados a la aplicación de la mecánica estadística, de modo que sus consideraciones posteriores sobre el átomo, que le llevaron a la creación de la mecánica de ondas, están basadas en sus primeros trabajos sobre la teoría estadística de los gases.

Boltzmann explicaba el comportamiento de los gases con la hipótesis atómica. Debido al hecho de que el gas estaba compuesto por muchísimos átomos, a los gases se les podía aplicar las leyes de la mecánica newtoniana con metodología estadística. Así Boltzmann mostró que los resultados obtenidos por métodos estadísticos, que implicaban necesariamente una supresión deliberada de muchísimos detalles, eran más interesantes que un tratamiento minucioso de todos estos, puesto que aportaban una nueva perspectiva a la situación física a la que se aplicaban. Asimismo (Schrödinger, 1935: 38) la prudente y sistemática ignorancia de los detalles llevaba a descubrir leyes de una nueva clase, que ofrecían nueva información sobre fenómenos naturales, humanos y sociales, de igual manera que las deducciones de la astronomía que se refieren al tamaño y la forma de las galaxias se derivan utilizando datos del número medio de estrellas. Así, para Schrödinger el método estadístico no solo constituía una característica dominante de su época sino también era un importante instrumento de progreso (Scott, 1967: 16).

En un principio los métodos estadísticos y el cálculo de probabilidad se habían utilizado solo para "remediar nuestros defectos, nuestra ignorancia de los detalles o nuestra incapacidad para enfrentarnos con el vasto material observacional” (Schrödinger, 1944: 704). Pero poco a poco la actitud ante el 
punto de vista estadístico había cambiado y el caso individual se había considerado completamente desprovisto de interés, tanto si se podía obtener información detallada de él como no, tanto si se podía disponer de un método matemático para enfrentarse a él como no ${ }^{8}$. Más drásticamente que cualquier otra ciencia, la física había promocionado el aspecto estadístico desde una función servil a una función dominante. Fue un paso revolucionario, que, según Schrödinger, afectó a todo el edificio de la ciencia, en cuanto que esta se halla basada en la física. Boltzmann dio este paso, que implicaba una nueva concepción de las leyes de la Naturaleza: estas no son leyes rigurosas, sino solo regularidades estadísticas, basadas en la ley de los grandes números.

Entre estas leyes naturales destaca la $2^{\text {a }}$ ley de la termodinámica, que enuncia el perpetuo incremento de la entropía y muestra la irreversibilidad que se presenta en el curso de la Naturaleza. Por esta ley, los hechos ocurren en una dirección definida, según la cual cada sistema evoluciona de forma natural desde un estado más ordenado a otro menos ordenado. Y esta ley, para Schrödinger,

Es la pura personificación de la ley estadística misma [...] Los hechos suceden en la dirección en que es más probable que ocurran. El calor fluye en la dirección en la que la temperatura cae, porque es billones de billones de veces menos probable que ocurra otra cosa (Schrödinger, 1944: 705).

${ }^{8}$ Según Schrödinger, el primer científico que fue consciente de la función relevante de la estadística fue Darwin, puesto que su teoría dependía de la ley de los grandes números. 
Un símil de esta tendencia natural (Schrödinger, 1967: 196) consiste en una baraja de cartas de juego que ordenamos. Si barajamos una, dos o tres veces esta baraja ordenada se convertirá en un conjunto desordenado de cartas. Pero puede pensarse también que, barajando el conjunto resultante, se consiga cancelar exactamente el efecto de las últimas operaciones y se restablezca el orden original. No obstante, todos esperamos que se produzca el primer proceso, mas no el segundo — en efecto, haría falta esperar mucho tiempo para que la baraja llegara, por azar, a ordenarse-.

Así, la regularidad que observamos en los procesos físicos ${ }^{9}$ es debida a la ley de la entropía. En todos los procesos se da una transición desde una situación atómica más ordenada a otra menos ordenada. En consecuencia, el balance total del desorden en la Naturaleza aumenta constantemente (Schrödinger, 1935: 39). De esta manera, la proposición fundamental del legado de Boltzmann es que la universalidad de la segunda ley de la termodinámica es en física macroscópica una consecuencia de la existencia de los átomos, de sus grandes cantidades y de unos adecuados supuestos estadísticos.

Esto tiene profundas implicaciones filosóficas. Nuestra comprensión de lo que ocurre en el mundo se reduce a razonamientos sobre grandes números. Podemos hacer predicciones porque podemos contar las posibilidades de cada hecho pensable y averiguar el que es más probable. "Que la probabilidad está, como regla, próxima a la certidumbre es debido al enorme número de

9 Según Schrödinger (Schrödinger, 1935: 196), la ley de la entropía gobierna todos los procesos físicos y químicos, incluso si forman parte de fenómenos que son tan complejos como la vida orgánica y el nacimiento y el desarrollo de las culturas humanas. 
átomos individuales o hechos microscópicos que cooperan" (Schrödinger, 1944: 706). La conexión existente entre estadística y atomística es evidente: si la estadística funciona y ayuda a entender el mundo es porque los cuerpos están compuestos de muchísimos átomos.

Así mostraba Schrödinger la herencia que había recibido de Boltzmann en su concepción general de los procesos físicos:

En todo proceso físico en el que observamos tal conformidad a la ley, intervienen innumerables millares —-más frecuentemente millares de millones— de átomos o moléculas [...] Al menos en un gran número de casos, de tipos absolutamente distintos, hemos logrado explicar la regularidad observada, como debida por completo al enorme número de procesos moleculares individuales que cooperan. El proceso individual puede tener o no su propia estricta regularidad: en la observada regularidad del fenómeno total no tiene que ahondarse en cada uno; por el contrario, queda absolutamente confundido entre millones de procesos análogos y los valores medios son los únicos observables para nosotros (Schrödinger, 1975: 17).

Exner, cuyos pensamientos estaban inspirados en el trabajo de Boltzmann, llevó más allá ${ }^{10}$ el punto de vista estadístico de este y planteó la cuestión de si toda la física puede tener una naturaleza estadística como consecuencia de su fundamento atomista —esta cuestión la encontramos también a menudo en Schrödinger-. Así manifestaba Exner:

${ }^{10}$ Según Jammer (Jammer, 1973: 587) el primer físico que cuestionó el estricto determinismo de las leyes físicas fue Boltzmann, aunque fue su discípulo Exner quien propuso claramente una concepción en la que los fenómenos macroscópicos constituyen el resultado de gran cantidad de procesos indeterministas. En cambio, en los escritos de Schrödinger aparece Exner como el primer físico que criticó el supuesto determinismo de los procesos atómicos. 
...cada suceso que se presenta en la Naturaleza es el resultado de hechos accidentales. Cuanto mayor es el número de hechos individuales de los cuales se compone un fenómeno, mayor es la probabilidad de que tenga un determinado carácter, mayor la probabilidad de que siga leyes definidas. Si la probabilidad de que un suceso ocurra de una determinada manera es tan elevada que, desde el punto de vista humano, se ha convertido en certeza, entonces hablamos de una "ley de la Naturaleza". Pero eso es posible solo si hay un número inimaginablemente grande de hechos individuales, como puede ocurrir en los procesos moleculares. En todos los otros campos, no hay leyes, solo regularidades y estas se hacen más dudosas cuanto menor es el número de hechos de los cuales son derivadas, hasta que finalmente, cuando el número se hace demasiado pequeño, se convierten en sucesos sometidos al azar (Moore, 1989: 153).

Exner no tenía evidencia empírica de estas ideas; las justificaba basándose en su creencia intuitiva de que la física se había de basar en una fundamentación unitaria. Por tanto, si las leyes a gran escala eran esencialmente estadísticas, Exner creía que las leyes a pequeña escala también lo tenían que $\operatorname{ser}^{11}$. La exposición del punto de vista de Exner fue el principal contenido de

${ }^{11}$ De esta forma, Exner mostraba, como ya había hecho Boltzmann, que con la noción de azar la mecánica estadística hace comprensibles las áreas a las que se aplica. Previamente el azar también había desempeñado un papel significativo para la comprensión de la Naturaleza en la teoría de Darwin. Así, según esta última teoría, el azar produjo la inteligencia que ha permitido entenderlo. Pero en "El futuro de la comprensión" (Schrödinger, 1967: 143), Schrödinger expone su preocupación por el modo de vida que prevalece en la sociedad, ya que, en lugar de promover una evolución que favorezca el progreso de la inteligencia, tiende a favorecer la "estupidización", la decadencia de esta facultad. Schrödinger advierte del peligro de que la mecanización y la estupidización de la mayoría de los procesos de trabajo contribuyan a que degenere nuestro órgano de la comprensión, nuestra inteligencia.

Naturaleza y Libertad. Número 6, 2016. ISSN: 2254-9668 
la lección inaugural de Schrödinger como profesor en la universidad de Zürich en 1922, titulada “¿Qué es una ley natural?”, en la que Schrödinger reconocía así la aportación de Exner:

Fue Franz Exner, un físico experimental, quien por primera vez, en 1919, con perfecta claridad filosófica, lanzó una crítica contra la manera como todo el mundo aceptaba, como algo dado por sentado, el determinismo absoluto de los procesos moleculares. Llegó a la conclusión de que aquello era ciertamente posible, pero de ninguna manera necesario y, examinado más de cerca, ni siquiera muy probable (Schrödinger, 1975: 22).

Schrödinger concluía su conferencia manifestando su esperanza de que, en un futuro, la concepción de Exner se impusiera en el mundo de la física teórica:

Yo prefiero creer que, una vez libres de nuestra enraizada predilección por la causalidad absoluta, lograremos superar estas dificultades y no que, a la inversa, la teoría atómica casi casualmente, diríamos - venga a comprobar el dogma de la causalidad (Schrödinger, 1975: 26).

La posibilidad de una fundamentación acausal de las regularidades de la Naturaleza permaneció, a lo largo de toda la vida de Schrödinger, como un tema central de sus reflexiones epistemológicas, como seguidamente vamos a ver.

\section{La causalidad y el azar}

Schrödinger reflexionó sobre la causalidad y el azar, como dos conceptos que ayudan a entender el mundo, el objetivo fundamental de la actividad 
científica. Ambas nociones desempeñan un papel central en la comprensión de la realidad, puesto que con ellas el científico pretende responder a la demanda de inteligibilidad que le presenta la exigencia de una imagen científica del mundo.

Schrödinger explicaba nuestra tácita y habitual aceptación del principio de la causalidad en nuestra vida cotidiana como resultado de un proceso adaptativo (Schrödinger, 1962: 15). El hombre se encuentra en un medio donde es consciente de que los cambios que éste presenta son muy importantes para su bienestar. La experiencia — tanto en la lucha de su vida diaria como en la lucha por el progreso en la investigación científica- le muestra que los procesos que se efectúan en su medio no suceden de forma arbitraria sino que presentan una notable regularidad; es decir, ciertos rasgos de los acontecimientos están siempre vinculados a otros. El ser humano trata entonces de captar la esencia de esta regularidad, pues comprende que su conocimiento le será muy útil. $Y$ entonces se da cuenta de que determinadas circunstancias que preceden a un fenómeno $A$ observado son invariables y, en cambio, otras son variables. Percibe que el grupo invariable siempre va seguido de $A$ y esto le hace afirmar que este conjunto constante de circunstancias es la causa de $A^{12}$. Y, más allá de la experiencia, el ser humano supone que aún en los casos en que no ha logrado encontrar la causa de un fenómeno también tiene que haberla. Este postulado que el hombre establece de forma inconsciente constituye el llamado principio de causalidad.

${ }^{12}$ Schrödinger escribe "la causa condicionante de A". 
Pero, si examinamos este principio con atención, como hizo Hume, vemos (Schrödinger, 1935: 33) que no podemos percibir ni comprender una conexión necesaria entre causa y efecto. La causación de un fenómeno por otro, como el calentamiento de la superficie de la tierra por el ascenso del sol, no se puede percibir directamente. Solo podemos percibir que un fenómeno, la subida del sol, siempre va seguido de otro fenómeno, el calentamiento de la superficie de la tierra. Vemos, a través de la experiencia, que en el mundo unos fenómenos suceden a otros, aunque no sea de forma evidentemente necesaria. Observamos que aparecen regularidades y tratamos de sintetizarlas a través de leyes científicas que pretendemos que sean universales.

Así vemos que en estas reflexiones Schrödinger seguía a Hume, cuya “cautelosa” (Schrödinger, 1962: 76) versión de la causalidad ${ }^{13}$ (Hume, 1977: 175) no era nada más que una inducción de la experiencia. La ciencia empírica se basa en la inducción. Pero ¿̇en qué se basa la inducción? Solo en la experiencia, en la observación de que en el pasado ha funcionado y ha sido útil, porque ha ayudado al ser humano en su lucha diaria. Mas, lógicamente, no se basa en nada. La inducción solo se basa, inductivamente, en el éxito que ha tenido en el pasado. Es, por tanto, un círculo vicioso.

La mecánica estadística, como hemos visto, también había planteado dudas sobre la existencia objetiva de las regularidades que expresan las leyes de la Naturaleza. La base de estas dudas es la inmensa cantidad de átomos y moléculas que participan en los procesos físicos cuya observación solo nos

${ }^{13}$ Porque Hume advierte que la suposición no siempre se cumple necesariamente, puesto que no tenemos impresión de la conexión necesaria entre causa y efecto. La visión de la causalidad que ofrece Hume le parece a Schrödinger la única "fidedigna” (Schrödinger, 1962: 76). 
permite extraer leyes estadísticas. Estas no siempre se cumplen con exactitud y a veces en los fenómenos se observan desviaciones de ellas, pero dichas diferencias presentan el carácter y la magnitud que la teoría estadística ya había calculado previamente. Un ejemplo de la naturaleza de las leyes estadísticas lo encontramos en las compañías de seguros (Schrödinger, 1935: 38). Los daños contra los que nos aseguramos — robos, incendios, accidentes- dependen de muchísimos factores y se muestran como fruto del azar. La compañía de seguros no distingue entre el tipo de perjuicio - si es un incendio, por ejemplo, o un robo- Solo le interesa el porcentaje de asegurados que van a sufrir un daño y van a tener que ser compensados. $Y$ este porcentaje puede ser previsto a partir de las regularidades estadísticas de los años anteriores. El azar está en la base de las regularidades. Así pues, del azar surge la ley.

\section{Dilema ante la cuestión de la causalidad de las leyes de la Naturaleza}

Frente a la cuestión de la causalidad de las leyes de la Naturaleza, a Schrödinger (Schrödinger, 1935: 40) se le presentaba un dilema, puesto que veía dos posibilidades. La primera consistía en aceptar que las leyes de la Naturaleza son de carácter estadístico y entonces el principio de causalidad no era, como mantenía Hume, lógicamente correcto sino que solo era un producto psicológico generado por el hábito de observar la regularidad estadística de los hechos - es decir, por inducción-. En el fondo, todos los fenómenos suceden por azar y no se sabe por qué suceden, porque el azar no está determinado por ninguna ley, no es explicable. Schrödinger se refería a esta concepción con estas palabras: 
La investigación física ha mostrado clara y definitivamente que el azar es, por lo menos en la abrumadora mayoría de los procesos naturales, la raíz de esa regularidad y de esa invariabilidad que nos han llevado a establecer el postulado de causalidad universal, en vista de su estricto ajuste a las leyes (Schrödinger, 1975: 17).

Así, las leyes de la Naturaleza surgen estadísticamente a partir de los fenómenos en que intervienen multitud de átomos que experimentan multitud de procesos que son casuales e inexplicables. Por esta razón, hay que eliminar de nuestra imagen científica del mundo la idea de la conexión causal entre fenómenos naturales. El azar está en la base de la Naturaleza y las regularidades causales que encontramos han surgido estadísticamente de la cooperación de miríadas de casualidades.

La segunda posibilidad consistía en no tener en cuenta la crítica de Hume al principio de causalidad y aceptar que dicho principio es a priori, es decir, es universal y es necesario para entender el mundo, porque, en el fondo, el mundo se rige por leyes de causa-efecto. Entonces pensaremos que, aunque no lo podamos observar, en realidad cada proceso se rige por una rígida causalidad. Así, Schrödinger se refería, en 1922, a esta concepción ${ }^{14}$ con las palabras siguientes:

La opinión general es que, en realidad, descubriríamos que el proceso individual — por ejemplo, la colisión de dos moléculas de gas-está determinado por una rígida causalidad, si pudiésemos seguirla, lo mismo que el resultado de una vuelta de la ruleta no sería cosa

${ }^{14} \mathrm{El}$ principio de causalidad era aceptado, en aquel momento, por la gran mayoría de físicos. Por esta razón Schrödinger no publicó su conferencia. 
del azar para quien conociese exactamente el ímpetu dado a la rueda, la resistencia del aire, la fricción del eje, etc. y supiese usar estos datos (Schrödinger, 1975: 22).

Si los fenómenos que observamos parecen fruto del azar es porque en ellos actúan innumerables elementos que son determinados por innumerables causas parciales que no somos capaces de observar. $\mathrm{El}$ azar es un término que usamos para reunir las numerosas causas que, aunque desconozcamos, sabemos que existen. Necesitamos los métodos estadísticos para encontrar leyes en la Naturaleza, pero esta, en el fondo, se rige por el principio de causalidad.

Ante este dilema, Schrödinger mantenía, en primer lugar, que el razonamiento igualmente nos lleva a inferir una alternativa como la otra, es decir, a concluir que el azar deriva de la ley y a concluir que la ley deriva del azar y, en segundo lugar, también mantenía que era difícil pensar que el dilema se pudiera decidir por vía empírica, a través de experimentos.

Durante años se había aceptado la segunda posición. La mecánica estadística no había abandonado la causalidad y el determinismo y la mayoría de físicos asumía que si las condiciones iniciales — por ejemplo, la posición y la velocidad - de cada molécula fueran completamente conocidas en un principio y se pudiera realizar un cálculo matemático exacto de todas las colisiones entre las moléculas, entonces se podría predecir lo que ocurriría. Pero si los físicos se habían de limitar a leyes estadísticas era por la imposibilidad práctica que tenían de conseguir tanto lo uno como lo otro (Schrödinger, 193: 35). Así se suponía que la naturaleza era, en esencia, determinista, es decir, que todos los fenómenos en el fondo estaban determinados por estrictas leyes causales. 
Como hemos visto, Exner ya había levantado sospechas sobre este fondo determinista y había planteado dudas sobre el supuesto aceptado de que los fenómenos en última instancia están determinados por precisas leyes causales. Lo había manifestado en 1919, es decir, unos años antes de que Heisenberg sacara a la luz su relación de indeterminación. Pero entonces no se había prestado atención a la reflexión de Exner. Y cuando Schrödinger, en su lección inaugural de 1922, la había defendido no había sido comprendido por el público ${ }^{15}$. Schrödinger, al final de su conferencia, aceptando las limitaciones del tratamiento que de un problema tan sumamente complejo había presentado, ${ }^{16}$ manifestaba:

La falta de tiempo me impide profundizar más en esta cuestión. He de limitarme a la siguiente observación general, que al mismo tiempo resume concisamente las conclusiones a las que hemos llegado.

${ }^{15}$ No fue hasta 1929 que Schrödinger se decidió a publicar su lección inaugural de 1922 con la siguiente nota introductoria: "Esta charla no fue publicada en su momento. La posterior aparición y desarrollo de la mecánica cuántica la ha colocado en el foco de interés, sin que, incidentalmente, el nombre de Exner haya sido mencionado”, según E. Schrödinger en Gesammelte Abhandlungen. Vol. 4 (p. 295).

16 Pero Schrödinger nunca encontró tiempo para elucidar sus ideas y reelaborar dicha conferencia en un ensayo filosófico más completo (Moore, 1989: 154). Por otra parte, a lo largo de los años trató en diversas ocasiones sobre este tema, pero nunca de forma sistemática, es decir, nunca dio forma definitiva a sus ideas al respecto y nunca se decidió por una postura única. Además, a menudo, encontramos en sus escritos una posición utilitaria, pues, tratando de problemas como el realismo y la causalidad, declara que un científico ha de usar las nociones que mejor le permitan conseguir su objetivo, que es acercarse a la Naturaleza. 
Causalidad, indeterminismo y libre albedrio

Lo que Exner afirmó equivale a esto: es perfectamente posible que las leyes de la naturaleza sean en conjunto de carácter estadístico. La ley absoluta que reside detrás de la ley estadística, y tenida por evidente en la actualidad por casi todo el mundo, va más allá del ámbito de la experiencia. Tal doble fundamento del curso ordenado de los acontecimientos en la naturaleza, es improbable en sí mismo. El peso de la demostración les toca a quienes defienden la causalidad absoluta, no a quienes dudan de ella. Una actitud de duda a este respecto es, hoy por hoy, la más natural (Schrödinger, 1975: 26).

La actitud de duda que era, según Schrödinger, la más justificada era debida a la mecánica cuántica que por principios, por razones teóricas, negaba la causalidad y el determinismo; especialmente por las relaciones de indeterminación de Heisenberg, que impiden conocer con precisión simultáneamente propiedades como la posición y la velocidad de una partícula; por tanto, como no se pueden conocer las condiciones iniciales en que se encuentra la partícula, tampoco se puede determinar su comportamiento posterior. Para Schrödinger, como para Heisenberg, la indeterminación de los valores iniciales impedía la predictibilidad de los valores futuros.

Pero, además, Schrödinger también presentaba otros argumentos. Uno de ellos surgía del carácter ondulatorio que él atribuía a la realidad. Según la mecánica ondulatoria el estado de un sistema físico queda determinado, unívocamente y sin ambigüedad, por el estado inicial para todo el porvenir; sin embargo, este estado y los que le siguen carecen de precisión, si se consideran desde el punto de vista clásico. Por ejemplo, consideremos un sistema simple, como un fotón o un electrón. Su estado inicial, su función $\Psi$, está representado por un tren de ondas que, si se encuentra un pequeño obstáculo, se transformará por difracción en una onda esférica que emana del obstáculo y se propagará sobre una parte del espacio cada vez mayor. Entonces se podría 
encontrar la partícula en diferentes direcciones, con diferentes probabilidades, que estarían determinadas por la intensidad que la onda esférica poseería en las diferentes direcciones. Esto significa que si se repitiera la experiencia, es decir, si se preparara la partícula de la misma manera antes de ser lanzada contra el obstáculo no produciría siempre el mismo efecto. Es lo que se conoce como defecto de igualdad, porque hay que "admitir que condiciones de estado inicial idénticas predisponen efectos diversos" (Schrödinger, 1996: 508).

Y otro argumento que también presentaba Schrödinger contra el determinismo era el siguiente: aun admitiendo la tesis determinista de que dado un conocimiento completo del estado de un sistema aislado se puede predecir exactamente su comportamiento futuro, hay que admitir que la Naturaleza es infinitamente complicada y por esta razón un sistema físico no puede ser determinado por un número finito de observaciones. Por tanto, todo lo que se le puede conceder al determinismo es que una infinita acumulación de observaciones permitiría determinar el sistema (Schrödinger, 1935: 64). Pero en la práctica solo se puede conseguir un número finito de observaciones.

Así, la mecánica cuántica había puesto de actualidad el debate sobre el determinismo y, según Schrödinger, había inclinado la balanza a favor del azar y el indeterminismo ${ }^{17}$, como anteriormente había propuesto Exner y posteriormente defendió la escuela de Copenhague. Sin embargo, Schrödinger es un autor poliédrico y también encontramos textos suyos donde muestra que se alejaba del indeterminismo y se acercaba a un cierto determi-

${ }^{17}$ Parece ser que por esta razón fue finalmente publicada la conferencia “¿Qué es una ley de la Naturaleza?" 
nismo, puesto que veía que la teoría cuántica "introducía nuevos principios de constricción, nuevos determinismos" (Arana, 2001: 31) que le llevaban a no creer que los procesos individuales fueran absolutamente casuales. Así, a pesar de las relaciones de indeterminación de Heisenberg y de la interpretación estadística de $\Psi$, se daba cuenta de que la teoría cuántica construía "leyes que condicionan con fuerza los casos individuales” (Arana, 2001: 32), como el principio de exclusión de Pauli.

\section{Indeterminismo y libre albedrío}

Por otra parte, la interpretación indeterminista de la mecánica cuántica, defendida por la escuela de Copenhague, había sido utilizada para defender el libre albedrío. Así, dicha interpretación había alimentado la esperanza de resolver este antiguo problema, la justificación de la libertad interior del ser humano, la explicación de por qué este se siente libre y responsable, explicación que es muy difícil si se considera al hombre como un ser determinado en un mundo completamente determinado. En cambio, en un mundo en que es impensable predecir el futuro parece más fácil entender la libertad interna y la responsabilidad del ser humano. Pero vamos a ver que Schrödinger (Schrödinger, 1936) consideraba que la esperanza de fundamentar el libre albedrío del hombre en el indeterminismo de la Naturaleza era una simple ilusión. Los argumentos que presentaba son los siguientes.

$\mathrm{Si}$ analizamos la situación vemos que pensamos de formas muy distintas cuando observamos a otras personas y cuando nos observamos a nosotros mismos. En el primer caso, si vemos que dos personas diferentes -o la misma persona en diferentes ocasiones- reaccionan de forma diferente en 
unas condiciones que, al menos aparentemente, son las mismas, tendemos a explicarlo por una real, aunque desconocida, diferencia de condiciones, en las que se incluyen las diferencias de carácter. Aquí no hay ningún indeterminismo. Simplemente somos como un físico determinista clásico que busca la causa de un fenómeno natural. La diferencia consiste en que aquí a la causalidad se la denomina motivación.

En cambio, en el segundo caso, cuando nos observamos a nosotros mismos, desempeñamos un papel muy diferente al del físico clásico determinista. Entonces nos sentimos auténticamente libres. $\mathrm{Y}$ en esta sensación de libre albedrío Schrödinger distingue dos rasgos diferentes. Por una parte, un primer rasgo constituye el hecho de que yo sé lo que voy a hacer, porque soy yo quien lo he decidido, quien quiere hacerlo. Y, por otra parte, el segundo rasgo característico del libre albedrío es el hecho de que yo me siento responsable de lo que voy a hacer, es decir, respondo de las consecuencias de mis actos. Antes de tomar mi decisión definitiva, antes de saber lo que voy a hacer, he contemplado diferentes posibilidades, entre las cuales tenía que elegir. Sentía que podía realizar varias acciones diferentes. Y esta posibilidad de elección ha sido interpretada por algunos como una realización en el ser humano del indeterminismo fundamental de la Naturaleza. Esta libertad para escoger mi actuación futura ha sido considerada la clave de la conexión del ser humano con la mecánica cuántica.

Otra clave para relacionar el libre albedrío y el indeterminismo cuántico la ha constituido el hecho de que el ser humano está compuesto de átomos. El argumento es muy sencillo. Las personas están formadas por átomos. Los átomos, en el fondo, se comportan de forma indeterminista. Por tanto, las 
personas también se comportan de forma indeterminista, es decir, tampoco están determinadas. En consecuencia, son libres.

Mas, según Schrödinger, la libertad del ser humano no tiene nada que ver con el indeterminismo cuántico. Lo muestra con un razonamiento al absurdo. Veámoslo. Si el libre albedrío se derivara del indeterminismo de la Naturaleza entonces ello implicaría dos consecuencias. Una consecuencia es que mis acciones habrían de seguir las relaciones de indeterminación de Heisenberg, que, por otra parte, yo podría alterar con mi decisión personal y mi fuerza de voluntad. Por ejemplo, si mi acción de fumar o no fumar antes del desayuno dependiera de dichas relaciones, éstas podrían predecir una probabilidad que yo podría invalidar con mi conducta. Es decir, si mis decisiones son regidas por las relaciones de indeterminación y yo puedo alterar mis decisiones ¿significa esto que yo puedo alterar dichas relaciones? Además, ¿si yo no hago lo que quiero sino lo que estipulan las relaciones de Heisenberg, por qué yo me siento responsable de mis actos?

Así, el supuesto de que el libre albedrío es, en el fondo, una aplicación del indeterminismo cuántico al ser humano implica consecuencias absurdas. Por tanto, Schrödinger no podía aceptar dicha suposición, por muy sugerente que en un principio pareciera.

Por otra parte, si nos observamos a nosotros mismos vemos que, según Schrödinger, nuestras verdaderas decisiones pueden tener diferentes motivaciones, pero no son tomadas por azar. Por esta razón nos sentimos responsables de ellas, porque cuando las tomamos nos sentimos justificados 
para tomarlas. En consecuencia, el libre albedrío nada tiene que ver con el indeterminismo cuántico ${ }^{18}$.

Cassirer había expuesto claramente esta conclusión, que Schrödinger compartía:

El libre albedrío del hombre conlleva, como factor preponderante, la conducta ética del hombre. Si suponemos que los hechos físicos en el espacio y el tiempo no están en gran medida estrictamente determinados y están del todo sujetos al azar, como cree la mayoría de los físicos de hoy, esta faceta aleatoria de los hechos en el mundo material sería indudablemente (dice Cassirer) la última en invocarse como correlato físico a la conducta ética del hombre. Porque, de hecho, lo es todo menos aleatoria; en realidad, está profundamente determinada por motivos que van desde los más viles hasta los más sublimes, desde la codicia y el despecho hasta el auténtico amor al prójimo o la sincera devoción religiosa (Schrödinger, 2009: 76).

\section{Condusiones}

Hemos visto que el problema del libre albedrío, del conflicto entre el determinismo de la Naturaleza y la libertad humana, surge al considerar que las causas de nuestros hechos psíquicos — pensamientos, sentimientos, deseos, decisiones...- son causas necesarias de las que el hombre no se puede escapar, como las causas propias de la concepción determinista, las causas que se corresponden unívocamente con sus efectos correspondientes.

${ }^{18}$ Desde otro punto de vista, desde la perspectiva biológica, hay que tener en cuenta, según Schrödinger (Schrödinger, 1936), que los procesos de la vida y de la consciencia son demasiado vastos y complejos para que en ellos se puedan percibir las relaciones de indeterminación de Heisenberg. 
La asociación entre causación y necesidad ya la había expresado claramente Spinoza en el axioma III del Libro I de su Ética: "Dada una determinada causa, el efecto se sigue por necesidad y, sin su causa, el efecto no se sigue". Esta supuesta asociación entre causa y necesidad era tan fuerte que, según Hume, se consideraba como una conexión lógica. Pero Hume la rompió al mostrar que, dada cualquier causa y su efecto correspondiente, no hay contradicción en suponer que la primera ocurre y la segunda no.

Pero la crítica de Hume reforzó la ecuación entre causalidad y necesidad, porque él mismo asumió (Hume, 1977: 181) que la conexión necesaria es un componente esencial de la relación entre causa y efecto. También Kant, al establecer la causalidad como un concepto a priori (Kant, 1976: 223), universal y necesario, reforzó la identidad entre causalidad y necesidad. Así, según Anscombe (Anscombe, 1971: 65) las aportaciones de ambos filósofos consolidaron la equivalencia ${ }^{19}$ entre el principio de causalidad y el determinismo.

Pero la causalidad no tiene por qué asociarse siempre con la necesidad ni con la generalización sin excepción, según la cual un hecho, considerado efecto, sigue inevitablemente otro hecho, considerado su causa. La causalidad, como propone Anscombe (Anscombe, 1971: 67), también puede entenderse simplemente como una derivación o procedencia del efecto a partir de la causa. Así, según esta concepción, que un hecho $B$ se derive o provenga de otro hecho $A$ no implica que cada hecho que sea como $B$ se derive o provenga de cada hecho que sea como $A$. Esta noción de causalidad se

${ }^{19}$ Como manifestaba Heisenberg, tal como hemos visto en el primer apartado de este trabajo. 
encuentra en el lenguaje común; por ejemplo, en el uso del término "contagio". Si contraigo una enfermedad infecciosa después de haber estado en contacto con una persona que padece o ha padecido dicha enfermedad decimos que el contacto ha sido causa del contagio; pero no decimos que siempre que haya contacto se producirá un contagio de la enfermedad; para que lo haya se han de dar otras condiciones.

En nuestro afán por conocerlo todo, tanto la res extensa como la res cogitans, buscamos de dónde provienen todos los fenómenos que percibimos, tanto los físicos como los psíquicos, es decir, buscamos los hechos anteriores de los que derivan los hechos que estudiamos. $\mathrm{Y}$ a estos hechos precedentes los consideramos causas. Quizás encontramos que algunas de estas causas son necesarias, es decir, están conectadas con sus efectos por leyes universales, sin ninguna excepción, que establecen correspondencias unívocas. Entonces decimos que al hecho $A$, la causa, le sigue siempre el hecho $B$, el efecto, o aplicamos la noción de probabilidad y decimos que a $A$ le sigue $B$ con una probabilidad del $100 \%$. Pero en la mayoría de nuestras indagaciones las causas que encontramos no son necesarias, no determinan inevitablemente los mismos efectos. Esto es lo que ocurre generalmente al investigar de dónde proceden los hechos psíquicos. Las causas que descubren las ciencias humanas y sociales no son causas necesarias, como las de los sistemas deterministas. Solo desvelan causas que influyen de formas diferentes en personas diferentes y en momentos diferentes, es decir, que condicionan el comportamiento de las personas, pero no lo determinan de forma unívoca ${ }^{20}$.

${ }^{20}$ Por ejemplo, vemos que se condiciona (se "castiga") a algunas personas para que no realicen una determinada acción y, a pesar de ello, igualmente la realizan 
El mundo psíquico es demasiado complejo para que, de momento, pueda caber en un sistema determinista.

Así pues, la mayoría de las causas condicionantes de nuestras decisiones que se han encontrado no constituyen una amenaza para nuestro libre albedrío. En el apartado anterior hemos visto que cuando Schrödinger examinaba el comportamiento de otras personas lo miraba desde fuera; entonces tenía la actitud del físico determinista. En cambio, cuando hacía introspección y contemplaba su propio comportamiento veía que al tomar sus propias decisiones se sentía libre y responsable de ellas. ¿Por qué? porque las personas vemos que podemos escoger entre diversas posibilidades de actuación. Hay factores que nos condicionan, pero en última instancia, nosotros podemos decidir cuál de las posibilidades queremos que se haga realidad. Schrödinger, como cualquiera de nosotros, también hubiera podido contemplar los factores condicionantes de sus decisiones personales desde fuera, hubiera podido buscar de qué hechos precedentes provenían, de qué causas se derivaban. Y podría haber descubierto algunas de ellas. Pero éstas, posiblemente, no hubieran sido causas necesarias, determinantes, solo condicionantes, porque en última instancia somos conscientes, en circunstancias normales, de que podemos cambiar nuestra decisión y hacer una elección diferente de la elección prevista como más probable.

En conclusión, hay campos de la res extensa donde funciona el modelo determinista — por ejemplo, el sistema solar-. Pero dicho modelo no se puede aplicar a todos sus dominios. Uno de ellos es el amplio campo de los procesos cuánticos, donde los físicos utilizan mayoritariamente un modelo 
indeterminista, aunque no para describir todos sus aspectos ${ }^{21}$. Tampoco funciona el modelo determinista en el amplio mundo de la res cogitans, donde nos encontramos con el libre albedrío. Mas la ciencia no ha podido mostrar conexión entre el indeterminismo de los procesos cuánticos y las causas condicionantes de los procesos psíquicos. Hay una inmensa distancia entre ambos dominios. Quizás esta distancia está constituida por una cantidad ingente de pasos intermedios que, de momento, en su mayoría, son desconocidos.

Mas sigue en pie la vieja aspiración intelectual de conocer las causas, de buscar los hechos anteriores de los que provienen los hechos - tanto físicos como psíquicos- que nos interesa entender, aunque estos no sean determinados necesariamente por aquellos, sino solo condicionados. Es más. Descubrir los factores condicionantes de nuestros pensamientos, afectos y decisiones nos ayuda a ser más libres, a disfrutar del estimado libre albedrío, es decir, nos acerca al anhelado ideal de la libertad, entendida ésta como el poder de escoger y realizar lo que nosotros realmente queremos ${ }^{22}$.

\section{Bibliografia empleada}

G.E.M. Anscombe, “Causality and Determination” (1971), en E.Sosa (ed.), Causation and Conditionals. Oxford University Press, 1975, pp. 63-81

\footnotetext{
${ }^{21}$ Así, en el tercer apartado hemos visto que Schrödinger mantenía el indeterminismo en unos aspectos y en otros no.

${ }^{22}$ Mas esta conclusión nos lleva a la difícil cuestión de qué entendemos con la noción de "libertad".
} 
J. Arana, Los filósofos y la libertad.Madrid, Síntesis, 2005

W. Heisenberg, "Atomphysik und Kausalgesetz"(1952), en Gesammelte Werke.Serie B. Berlin,Springer Verlag, 1985

D. Hume, Tratado de la Naturaleza Humana. Madrid. Editora Nacional, 1977

M. Jammer, "Indeterminacy in Physics", en Dictionary of the History of Ideas. New York, Charles Scribner's Sons, 1973, vol.2, pp. 586-593, disponible on line

I. Kant, Crítica de la razón pura. Buenos Aires, Losada, 1976.

W. Moore, Schrödinger, life and thought.Cambridge, Cambridge University Press, 1989

E. Schrödinger, Gesammelte Abhandlungen, Wien, Österreichische Akademie der Wissenschaften, 1984.

—.”The meaning of wave mechanics”, en Gesammelte Abhandlungen, Wien, Österrichische Akademie der Wissenschaften, 1984

—. "Indeterminism and Free Will", en Nature, 1936, July 4

—. "La nueva mecánica ondulatoria" (1927), en Heisenberg. Bohr. Schrödinger. Física cuántica. Barcelona. Biblioteca Universal del Círculo de Lectores, 1996

-. ¿Qué es una ley de la Naturaleza? (1962), México, FCE, 1975.

- Science and the Human Temperament, London, G. Allen \& Unwin Ltd., 1935, disponible on line

-. Ciencia y Humanismo (1951), Barcelona, Tusquets, 2009

-. Què és la vida? La ment i la matèria (1956), Barcelona, Eds. 62, 1967

—. "The statistical law in Nature", en Nature, 1944, vol. 153, pp. 704

- "The present situation in Quantum Mechanics"(1935) en J. A.Wheeler y W. H.

Zurek (Editores), Quantum Theory and Measurement.Princeton, Princeton University Press, 1983, pp. 152-167

- La nueva mecánica ondulatoria y otros escritos. Edición e introducción de J.Arana. Madrid, Biblioteca Nueva, 2001

W. T. Scott, Erwin Schrödinger. An Introduction to His Writings. Massachusetts, Massachusets Press, 1967

B. Spinoza, Ética.Madrid, Alianza, 2011

Josefa Castellà Cid

jcastellacid@hotmail.com

Naturaleza y Libertad. Número 6, 2016. ISSN: 2254-9668 